\title{
Interfacial debonding of a multi-cracked and coated hollow cylinder due to convective heating
}

\author{
Xuejun Chen ${ }^{\mathrm{a}, \mathrm{b}, *}$, Kun Zhang ${ }^{\mathrm{a}}$, Guangnan Chen ${ }^{\mathrm{a}}$, Gengxing Luo ${ }^{\mathrm{a}}$ \\ ${ }^{a}$ Laboratory of Surface Modification, Institute of Mechanics, Chinese Academy of Sciences, No. 15 Beisihuanxi Road, Beijing 100080, China \\ ${ }^{\mathrm{b}}$ Graduate School of the Chinese Academy of Sciences, Beijing 100080, China
}

Received 20 December 2005; accepted in revised form 22 December 2005

Available online 9 February 2006

\begin{abstract}
The interfacial debonding of an inner-surface-coated hollow cylinder with multiple pre-existing surface cracks is presented. The cylinder is assumed to be exposed to convective heating at the inner surface while the outer surface is perfectly insulated. The failure criterion based on the adhesion strength is adopted, in which the interfacial stresses are considered to be responsible for interfacial debonding initiation. By using the finite element (FE) method, the transient temperature and induced interfacial stresses are calculated for the cylinder. Based on dimensional analysis, the sensitivity analysis of interfacial stresses is obtained as a function of various dimensionless parameters such as time, convection severity, material constants, crack depth as well as crack spacing. These results may provide some useful references for coating/substrate system design under thermal loading.
\end{abstract}

(C) 2006 Elsevier B.V. All rights reserved.

Keywords: Coated hollow cylinders; Convective heating; Multiple surface cracks; Dimensional analysis; Coating debonding

\section{Introduction}

The thermally induced failure problem of thick-walled cylinders has been widely investigated, due to its importance to the design and manufacturing of such structural components as the pressure vessels, piping subjected to thermal transients [1-6]. In many modern applications, coatings are intentionally deposited on the underlying substrate to enhance some specific surface characteristics, e.g. strength, corrosion and wear resistance. Since the durability of a coated hollow cylinder is largely dependent on the life of coatings, failure mechanisms of coatings under thermal loading have been the subject of considerable research [7-12]. In general, coating debonding along the interface is considered to be most dangerous since it will lead to fatal failure of the entire thermo-mechanical system $[11,12]$.

\footnotetext{
* Corresponding author. Laboratory of Surface Modification, Institute of Mechanics, Chinese Academy of Sciences, No. 15 Beisihuanxi Road, Beijing 100080, China. Tel.: +86 $1062547527 x 5$; fax: +86 1062547526 .

E-mail address: chenxuejuncas@sohu.com (X. Chen).
}

Previous studies on interfacial debonding were mainly from the well-established standpoint of interface crack growth, in which an interfacial crack is assumed to already exist $[13,14]$. This model seems sound and justified for the interface of relatively weak adhesion strength, where an initial separation could occur even before service. For the interface of strong adhesion strength, however, a reasonable attention should be paid on the intact interface. In this case tensile or shear stress at the interface must serve as a prerequisite for the interfacial debonding initiation. Therefore the quantification and sensitivity analysis of these stresses are the main tasks of this paper. In practice, surface cracks commonly occur in the coating due to strain mismatch of coating/substrate system after the deposition is completed [15]. Recently a processing technique, discrete laser quenching on the inner surface of gun tubes prior to the electroplation of chromium, was successfully developed to enhance the durability of chromium on the steel substrate. Field testing showed that the treated gun tubes could survive longer than the untreated ones $[16,17]$. The essence of this processing technique is considered to be connected to the periodic segmentation of coatings, which is in favor of strain accommodation. Hence multiple surface cracks are also 
included in the subsequent model to capture this realistic situation.

In the present paper, the problem of a multi-cracked and coated hollow cylinder, subjected to convective heating at the inner surface is considered. In solving the problem, it is assumed that both coating and substrate materials are homogeneous, isotropic and linear-elastic, the thermo-mechanical constants are independent of temperature, the thermoelastic coupling effects and inertia effects are negligible. Previous work on dynamic thermoelasticity indicated that the last assumption, which simplifies the analysis of the problem quite considerably, would not cause any significant changes in the results $[18,19]$. All the calculations are completed via the finite element (FE) method. Numerical results for temperature and interfacial stresses are obtained as a function of normalized quantities such as time, convection severity, material constants, crack length as well as crack spacing.

\section{Problem formulation}

For a finitely long cylinder, the stress analyses are usually 3D problems. It is modeled here as a plane strain problem. This simplification is considered to represent a reasonable idealization for the stresses and displacements in the central portion of a long cylinder to provide useful bounds. The geometry of a multi-cracked and inner-coated hollow cylinder, with cylindrical coordinates $(r, \phi, z)$ embedded at its center, is shown in Fig. 1a. Its inner radius, outer radius and coating thickness are denoted by $a, b$ and $h$, respectively. $n$ uniformly spaced radial cracks of depth $l$ (only four cracks are plotted in Fig. 1) are contained in the coating. The cylinder, at an initial temperature $T_{0}$, is assumed to be perfectly insulated along the outside surface $r=b$. At time $t=0$, the inner surface $r=a-h$ is subjected to convective heating with the heat transfer coefficient given by $H$ and the ambient temperature maintained at $T_{\mathrm{a}}$.

The existence of multiple radial cracks would not cause any disturbance in temperature field throughout the coated hollow cylinder. By nature of axis-symmetry, the transient temperature is largely simplified to a 1-D thermal diffusion problem, which may be determined from the solution of the following diffusion equations

$\Delta^{2} \theta_{i}(r, t)=\frac{1}{D_{i}} \frac{\partial \theta_{i}(r, t)}{\partial t} \quad(i=1,2)$

where temperature differences $\theta_{i}$ are defined by

$\theta_{i}(r, t)=T_{i}(r, t)-T_{0} \quad(i=1,2)$

$\theta_{0}=T_{\mathrm{a}}-T_{0}$

The subscripts 1,2 represent coating and substrate, respectively. The thermal diffusivity $D_{i}$ is given by $D_{i}=k_{i} / \rho_{i} c_{i}$, where $k_{i}$ is the material thermal conductivity, $\rho_{i}$ the density and $c_{i}$ the specific heat. In the case of convective heating, $\theta_{0}>0$.

The initial conditions are given by

$\theta_{1}(r, 0)=0 \quad(a-h<r<a) \quad \theta_{2}(r, 0)=0 \quad(a<r<b)$.

The continuity and boundary conditions are usually written as

$\theta_{1}(a, t)=\theta_{2}(a, t), \quad k_{1} \frac{\partial}{\partial r} \theta_{1}(a, t)=k_{2} \frac{\partial}{\partial r} \theta_{2}(a, t) \quad(t>0)$

$\frac{\partial}{\partial r} \theta_{1}(a-h, t)=\frac{H}{k_{1}}\left[\theta_{1}(a-h, t)-\theta_{0}\right], \quad \frac{\partial}{\partial r} \theta_{2}(b, t)=0$

It is emphasized that Eq. (4a) indicates the continuity of temperature and heat flux at the coating/substrate interface, neglecting contact thermal resistance.

When the thermal stresses are considered, the axis-symmetry breaks. However, a representative sector, with the central angle of $\pi / n$, can be extracted for analysis as shown in Fig. 1b. (a)

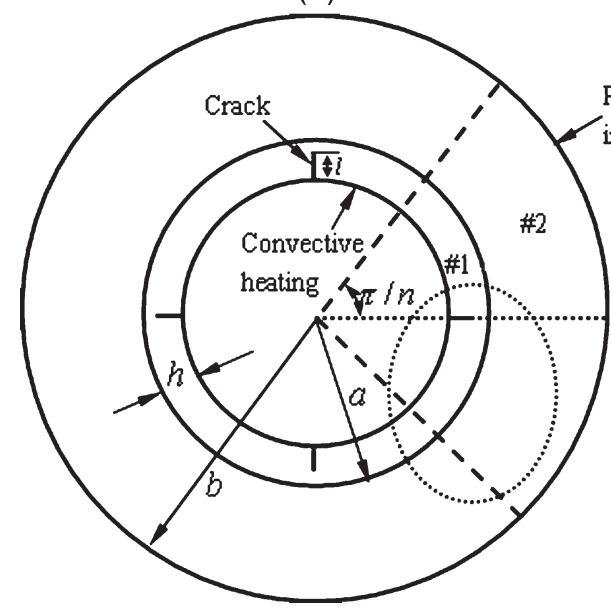

(b)

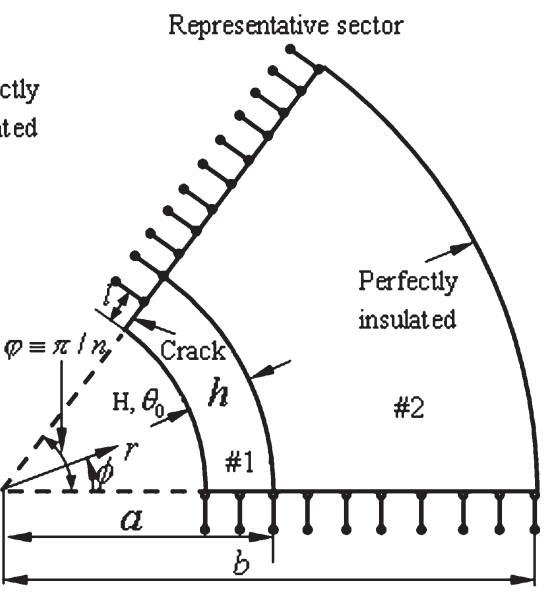

Fig. 1. (a) The multi-cracked coated cylinder subjected to convective heating from inner surface; (b) the geometry of an extracted representative sector with imposed boundary conditions. 
Bearing in mind the usual equilibrium, constitutive and geometrical equations, which can be easily found in any thermo-elasticity textbook, we write the following boundary and continuity conditions for thermal stress calculations

$$
\begin{aligned}
& \sigma_{r r 1}(a-h, \phi)=\sigma_{r \phi 1}(a-h, \phi)=0 ; \sigma_{r r 2}(b, \phi)=\sigma_{r \phi 2}(b, \phi) \\
& =0 \quad(0<\phi<\pi / n) \\
& u_{r 1}(a, \phi)=u_{r 2}(a, \phi) ; u_{\phi 1}(a, \phi)=u_{\phi 2}(a, \phi) \quad(0<\phi<\pi / n)
\end{aligned}
$$

$\sigma_{r r 1}(a, \phi)=\sigma_{r r 2}(a, \phi) ; \sigma_{r \phi 1}(a, \phi)=\sigma_{r \phi 2}(a, \phi) \quad(0<\phi<\pi / n)$

Where $u_{\mathrm{r}}$ is the radial displacement, $u_{\phi}$ the circumferential displacement, $\sigma_{r r}$ the radial normal stress, $\sigma_{\phi \phi}$ the circumferential normal stress and $\sigma_{r \phi}$ the shear stress. In order to account for coating debonding, emphasis is solely placed on interfacial stresses in subsequent thermal stresses calculation.

\section{Solution procedures}

\subsection{Dimensional analysis}

Before the solution to the above problem is known, dimensional analysis can be carried out to reveal the scaling relationships of this problem. From Eqs. (1) (2a,b) (3) (4a,b), the temperature differences $\theta_{i}(i=1,2)$ must be functions of all the independent governing parameters, i.e., temporal and spatial quantities $t$ and $r$; characteristic geometrical parameters $a, b$ and $h$; thermo-physical constants $D_{1}, D_{2}, k_{1}, k_{2}, H$ and ambient temperature difference $\theta_{0}$

$\theta_{i}(r, t)=f\left(r, t ; a, h, b ; D_{1}, D_{2}, k_{1}, k_{2} ; H, \theta_{0}\right)$

Among the twelve governing parameters, four of them, namely $h, D_{1}, k_{1}$ and $\theta_{0}$, have independent dimensions. The dimensions of $\theta_{i}, r, t, a, b, D_{2}, k_{2}$ and $H$ are given by

$$
\begin{aligned}
& {\left[\theta_{i}\right]=\left[\theta_{0}\right] \quad[r]=[h] \quad[t]=\left[D_{1}\right]^{-1}[h]^{2} \quad[a]=[h]} \\
& {[b]=[h] \quad\left[D_{2}\right]=\left[D_{1}\right] \quad\left[k_{2}\right]=\left[k_{1}\right] \quad[H]=\left[k_{1}\right][h]^{-1} .}
\end{aligned}
$$

On applying $\Pi$ - theorem in dimensional analysis [20], we obtain

$$
\frac{\theta_{i}}{\theta_{0}}=\Pi_{\phi}\left(\frac{r}{h}, \frac{D_{1} t}{h^{2}} ; \frac{a}{h}, \frac{b}{h} ; \frac{D_{2}}{D_{1}}, \frac{k_{2}}{k_{1}}, \frac{H h}{k_{1}}\right)
$$

The following dimensionless quantities are then naturally defined as

$\bar{r}=\frac{r}{h} \quad \bar{a}=\frac{a}{h} \quad \bar{b}=\frac{b}{h} \quad \bar{D}=\frac{D_{2}}{D_{1}}$

$\bar{k}=\frac{k_{2}}{k_{1}} \quad \mathrm{Bi}=\frac{H h}{k_{1}} \quad \mathrm{Fo}=\frac{D_{1} t}{h^{2}}$.

Based on the above dimensional analysis, the dimensionless quantity $\theta_{i} / \theta_{0}$ on the left side of Eq. (8) is determined only by seven bracketed dimensionless quantities on the right side, in which $\mathrm{Bi}$ and $\mathrm{Fo}$ are similar to conventional Biot number and Fourier number, respectively. The most severe thermal shock case corresponds to a sudden heating, i.e., $\mathrm{Bi} \rightarrow \infty$.

Similarly, thermal stress components depend on the following quantities

$\sigma(r, \phi, t)=f\left(r, \phi, t ; \theta_{0}, H, D_{1}, D_{2}, k_{1}, k_{2} ; a, b, h, l, \varphi ;\right.$

$\left.E_{1}, E_{2}, v_{1}, v_{2}, \alpha_{1}, \alpha_{2}\right)$.

On applying $\Pi$ - theorem again, we obtain

$\frac{\left(1-v_{1}\right) \sigma}{E_{1} \alpha_{1} \theta_{0}}=\Pi_{\sigma}\left(\frac{r}{h}, \frac{\phi}{\phi}, \frac{t D_{1}}{h^{2}} ; \frac{H h}{k_{1}}, \frac{D_{2}}{D_{1}}, \frac{k_{2}}{k_{1}} ; \frac{a}{h}, \frac{b}{h}, \frac{l}{h} ; \frac{E_{2}}{E_{1}}, \frac{v_{2}}{v_{1}}, \frac{\alpha_{2}}{\alpha_{1}}\right)$.

As can be shown by Eq. (11), three additional dimensionless material properties, $\bar{E}=E_{2} / E_{1}, \bar{v}=v_{2} / v_{1}$ and $\bar{\alpha}=\alpha_{2} / \alpha_{1}$ should appear automatically in thermal stress analysis, which measure the ratios of Young's modulus, Poisson's ratio and thermal expansion coefficient (CTE), respectively. In addition, dimensionless geometrical parameters $\bar{\phi}=\phi / \varphi$ and $\bar{l}=l / h$ also come out to account for the crack related configuration. With the help of the above dimensional analysis, sensitivity analysis for temperature, thermal stresses can be conveniently performed, which will be detailed in Section 4 .

\subsection{Finite element model}

In fact, there exists a closed form solution for the temperature in the above cylinder, in the form of infinite series of Bessel functions and Neumann functions [5]. For convenience, both temperature and thermal stresses calculation in this paper are completed via the finite element (FE) method. As mentioned in Section 2, only a sector of the coated hollow cylinder, with a central angle of $\pi / n$, has to be considered. In order to obtain accurate results, careful element meshing is necessary to assure a reasonable aspect ratio of the elements, and particularly element refinements should be done in the vicinity of the crack tip and the interface of coating and substrate.

Eight-node isoparametric quadrilateral elements are used here for both temperature and displacement calculations. The detailed mesh breakdowns are schematically illustrated in Fig. 2. The associated boundary conditions should be understood as follows: (i) For the temperature calculation, perfect insulation $(\mathrm{d} T / \mathrm{d} n=0)$ is imposed on the circumferential direction along the entire radial lines; (ii) For the thermal stress 


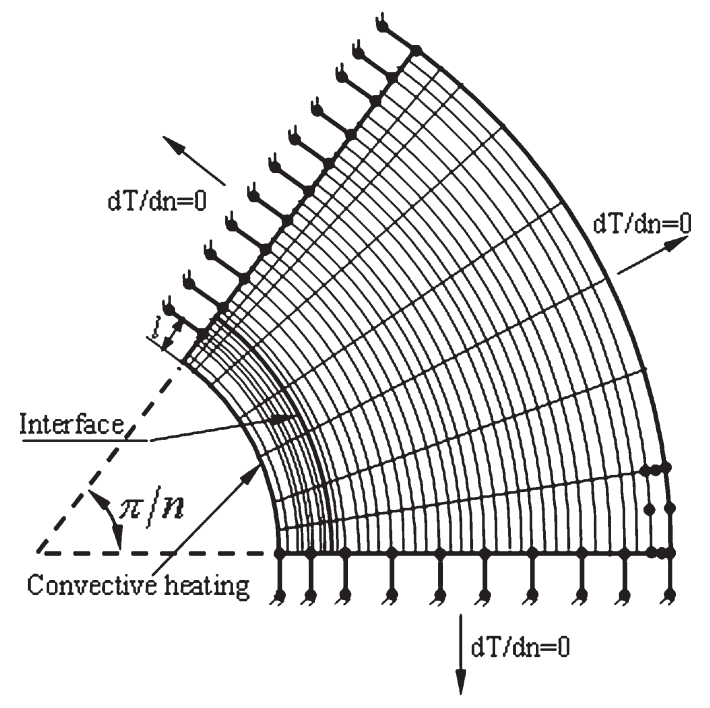

Fig. 2. Schematic finite element meshes and boundary conditions in the analysis.

calculation, the crack line is free while circumferential displacement of the remaining radial line vanishes. With the temperature distribution in the coated cylinder known, it is easy to determine the transient thermal stress by the classical finite element formulation. By endowing the coating and substrate with the same material constants and letting $\mathrm{Bi} \rightarrow \infty$, a series of runs were performed to duplicate various transient temperature results obtained by Tang and Erdogan [5], most of which were found to be in excellent agreement with those in references. Hence the present FE model is reasonably validated.

\section{Numerical results and discussions}

As an example for the convective heating problem described in the previous sections, we consider a material pair, which corresponds to a chromium coating deposited onto a much thicker structural steel substrate 30CrNi2MoVA [21]. Since the problem is formulated in terms of dimensionless quantities, it is sufficient to consider only the ratios shown in Table 1. Unless otherwise stated, we have taken data from Table 1 in our calculations whenever the required input quantities are not displayed. All the quantities of interest are normalized in the same forms as those in Section 3.1.

\subsection{Results of transient temperature}

Fig. 3 illustrates the normalized temperature versus normalized time at three different locations: (A) the inner surface, (B) the interface and (C) the substrate which is one coating thickness below the interface. It is shown that, temperature at all three locations first climbs sharply then the rising pace becomes slower with the elapse of time. Until

Table 1

The dimensionless quantities used in the example [21]

\begin{tabular}{lllllll}
\hline$a / h$ & $\mathrm{~b} / h$ & $D_{2} / D_{1}$ & $k_{2} / k_{1}$ & $E_{2} / E_{1}$ & $v_{2} / v_{1}$ & $\alpha_{2} / \alpha_{1}$ \\
\hline 100 & 200 & 0.293 & 0.389 & 0.724 & 1.476 & 1.923 \\
\hline
\end{tabular}

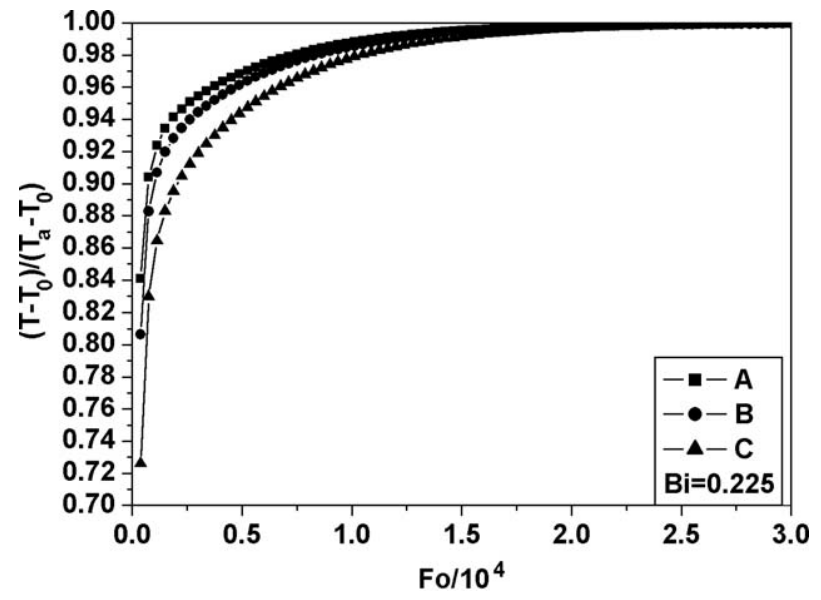

Fig. 3. The normalized temperature versus normalized time for various locations, $\mathrm{Bi}=0.225$. Other inputs are listed in Table 1 . Points $\mathrm{A}, \mathrm{B}, \mathrm{C}$ are located at the inner surface, interface and one coating thickness below the interface, respectively.

steady state is reached, the temperature at the inner surface remains highest. For the present data set, the steady state is arrived when Fo approaches 30,000.

The transient temperature distributions in the coated hollow cylinder are shown in Figs. 4 and 5. At various time instants, the variation of normalized temperature versus normalized radial coordinate is plotted in Fig. 4. It is shown that, the temperature intractably increases with time and such a trend constantly penetrates into the coated cylinder. The temperature gradient within the coating is a decreasing function of time until steady state is reached. At the coating/substrate interface, the slope of curve discontinues, indicating the different thermal resistances of coating and substrate. It should be noted in passing that, since $k_{2} / k_{1}=0.389$, the chromium coating does not act as a thermal barrier coating. In order to investigate the effect of convection severity on the normalized temperature distribution, different Biot numbers were chosen at early time Fo $=30$ in Fig. 5 to find that, the increase of Biot number could cause significant increase of the normalized temperature throughout the coated hollow

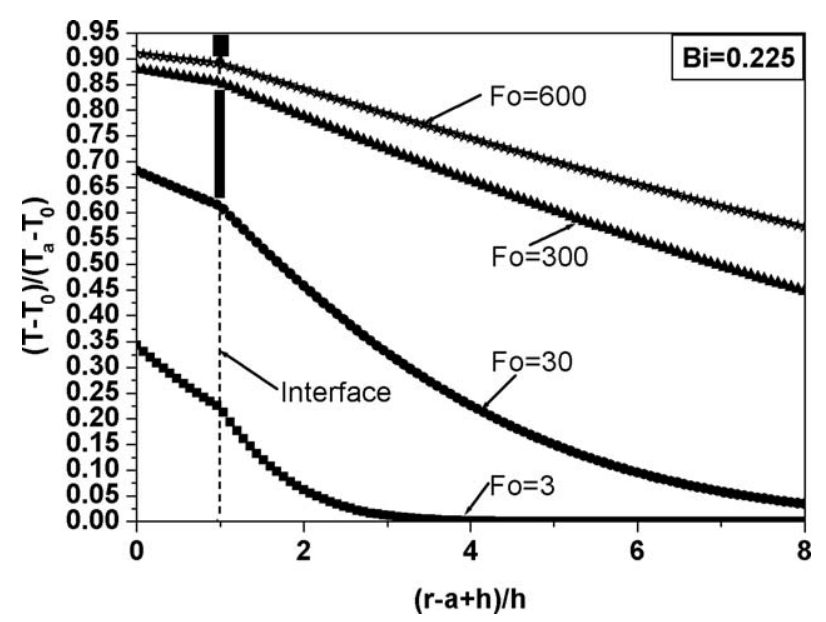

Fig. 4. The normalized transient temperature versus normalized radial coordinate for various time instants, $\mathrm{Bi}=0.225$. Other inputs are listed in Table 1. 


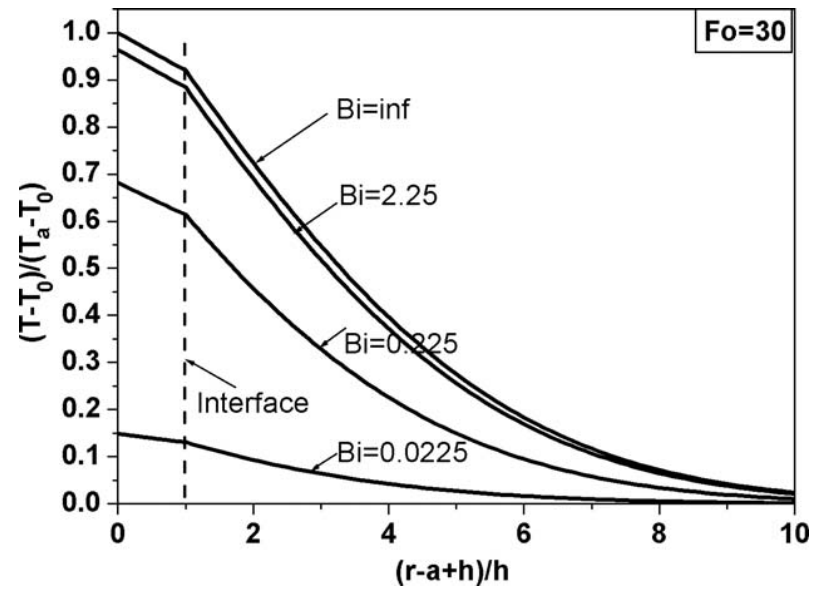

Fig. 5. The normalized transient temperature versus normalized radial coordinate for various Biot numbers, Fo $=30$. "inf" stands for " $\infty$ ". Other inputs are listed in Table 1.

cylinder. However, the increasing rate of normalized temperature becomes lower with further increase of convection severity.

\subsection{Results of interfacial thermal stresses}

The variation of interfacial stress components in the vicinity of steady state versus normalized angle $\phi / \varphi$ for crack number $n=30$ is plotted in Fig. 6. It is shown that near the crack tip, there exists very high tensile interfacial normal stress. In overview, the sign of interfacial normal stress changes twice over the entire interfacial arc: when interfacial point becomes more away from the crack tip, the tensile stress first decays sharply then is converted into compression at the point about $16 \%$ remote from the crack tip. This shift law is consistent with the self-equilibrating nature of thermal stresses. In addition, compared to the shear stress component, the normal stress component near the crack tip is prominent and therefore is considered to be the dominant driving force for interfacial debonding. In Fig. 7 the variation of transient interfacial stress near the crack tip versus normalized time is plotted. This plot shows that the profile of interfacial stresses history is like a

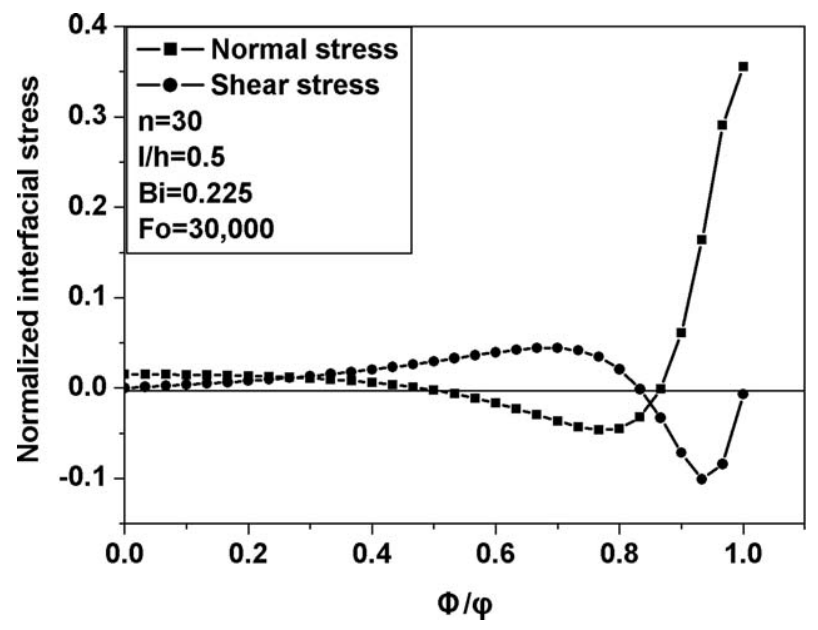

Fig. 6. The normalized interfacial normal stress versus normalized angle $\phi / \varphi$. $n=30, l / h=0.5, \mathrm{Bi}=0.225, \mathrm{Fo}=30,000$. Other inputs are listed in Table 1 .

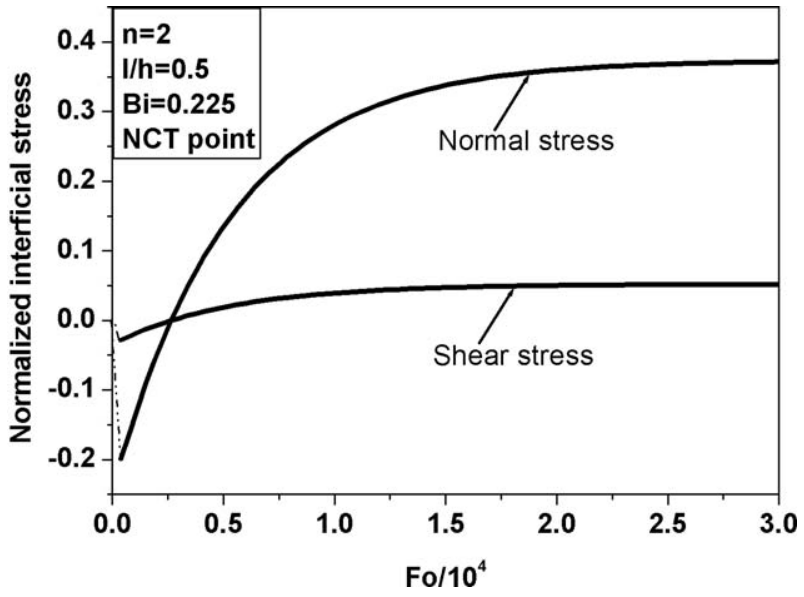

Fig. 7. The normalized interfacial normal stress near the crack tip versus normalized time. $n=2, l / h=0.5, \mathrm{Bi}=0.225$. Other inputs are listed in Table 1 .

"scoop". At early time interfacial stress components take on compressive as the result of constraint imposed by the relatively cooler substrate; after reaching the negative, peak value it gradually decreases and is converted into positive then remains constant up to the steady state. From Figs. 6 and 7, one can conclude that for the present data set, the largest interfacial normal stress occurs near the crack tip (NCT), $\phi / \varphi=1$, when steady state is approached. Hence in subsequent sensitivity analysis, focus is solely on the interfacial normal stress of NCT near the steady state.

\subsection{Sensitivity analysis of interfacial normal stresses of the NCT point}

Fig. 8 demonstrates the effect of convection severity on the normalized NCT interfacial normal thermal stress. It is shown the NCT interfacial normal thermal stress increases with the increase of Biot number prior to the steady state, while the ultimate value is almost not influenced by the convection severity. This implies that the more severe the convection heating, the earlier the coating debonding will occur. It is shown in Fig. 9 that, the normalized NCT interfacial normal stress is

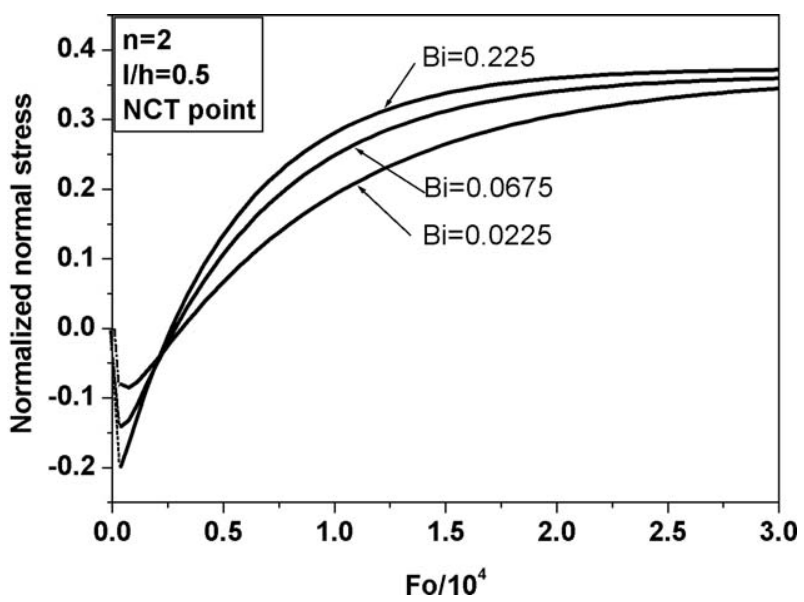

Fig. 8. The normalized NCT interfacial normal stress versus normalized time for various Biot numbers. $n=2, l / h=0.5$. Other inputs are listed in Table 1 . 


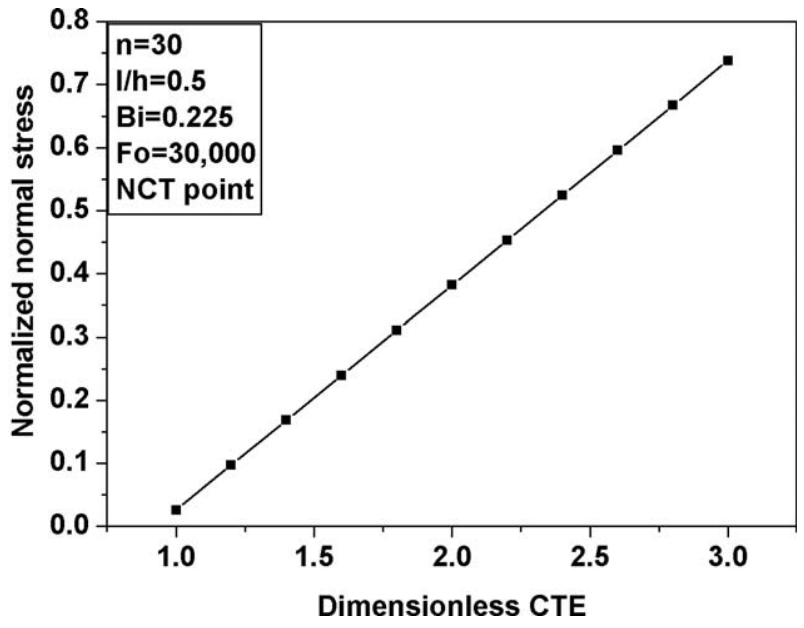

Fig. 9. The normalized NCT interfacial normal stress versus dimensionless CTE. $n=30, l / h=0.5, \mathrm{Bi}=0.225, \mathrm{Fo}=30,000$. Other inputs are listed in Table 1 .

almost a linearly increasing function of normalized ratio of coefficients of thermal expansion $\bar{\alpha}=\alpha_{2} / \alpha_{1}$. This indicates for a given substrate the increase of coefficient of thermal expansion (CTE) of coating may lead to fewer tendencies of interfacial debonding. Fig. 10 illustrates the effect of normalized Young's modulus on the normalized NCT interfacial normal stress, indicating the increase of it can cause the increase of normalized interfacial stress and the possibility of interfacial debonding. This effect is in consistent with fact that a more compliant coating is more prone to decohesion, previously validated by Hutchinson and Suo [13]. However, it would not seem so notable upon further increase of modulus ratio.

Fig. 11 shows the effect of normalized crack depth on normalized NCT interfacial normal stress. It is shown the NCT interfacial normal thermal stress increases with the increase of crack depth. This effect is due to the fact that, when the crack tip penetrates further near the NCT point, the NCT point can feel higher intensity of the crack-induced stress concentration. The variation of the normalized NCT interfacial normal stress versus the central angle of the representative sector is plotted in Fig. 12.

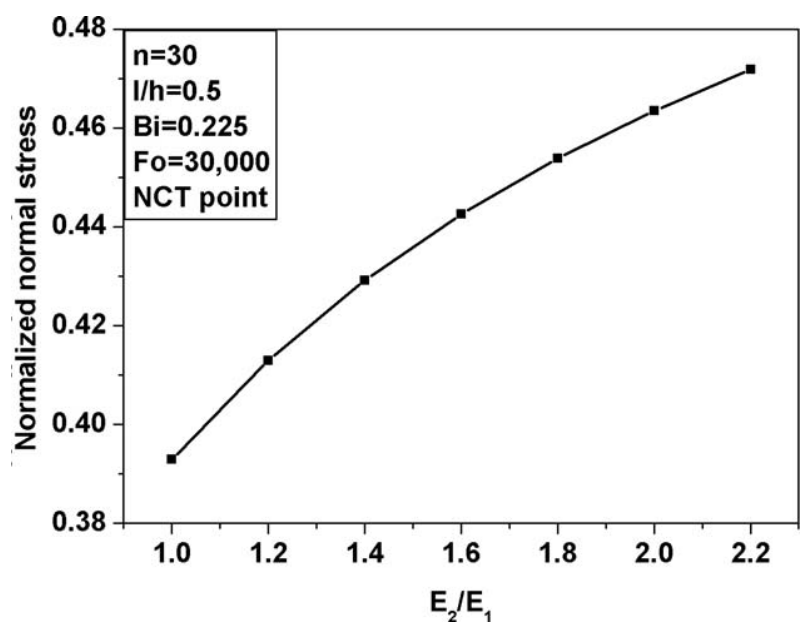

Fig. 10. The normalized NCT interfacial normal stress versus normalized Young's modulus. $n=30, l / h=0.5, \mathrm{Bi}=0.225, \mathrm{Fo}=30,000$. Other inputs are listed in Table 1.

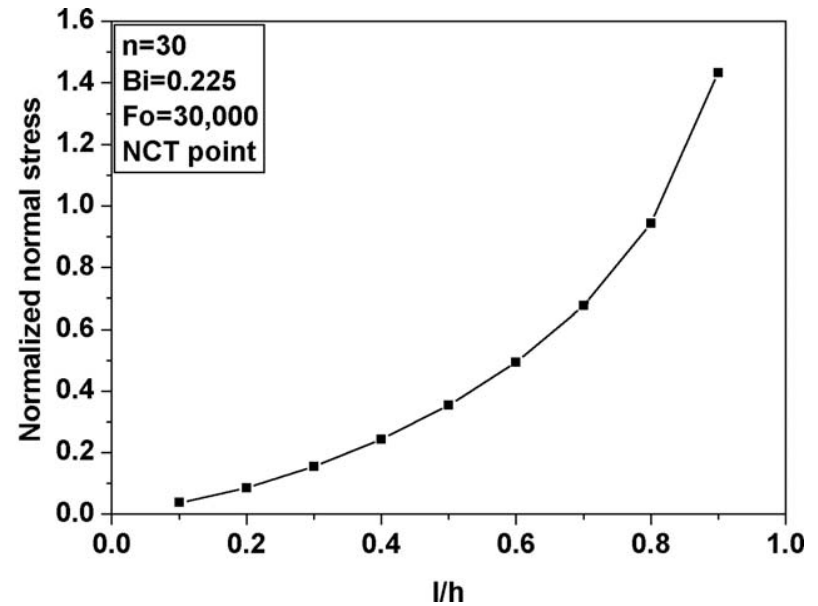

Fig. 11. The normalized NCT interfacial normal stress versus normalized crack depth. $n=30, \mathrm{Bi}=0.225, \mathrm{Fo}=30,000$. Other inputs are listed in Table 1 .

Since the central angle is inversely related to the crack number, the graph actually represents the dependence of interfacial debonding on the surface cracking. It is shown that for a given normalized crack depth, the magnitude of normalized NCT interfacial normal stress first decreases slightly with the increase of crack number, followed by a sharp decrease with further increase of surface cracking. This variation trend can be understood as follows: when crack spacing is very large with small crack number, the interaction between multiple cracks is little; when crack spacing becomes smaller and smaller, the redistribution of thermal stress induced by the introduction of multiple cracks generates "shedding effect", causing the decrease of interfacial normal stress in the vicinity of the crack tip. Thus, by multiple segmentations of coatings durability of coating can be greatly enhanced. This interesting and important phenomenon has been experimentally proven for planar interfacial debonding of thermal barrier coatings (TBC) in previous literature [22-24]. It can also be applied to interpret the field testing results of discrete laser processing technique for coated gun tubes $[16,17]$.

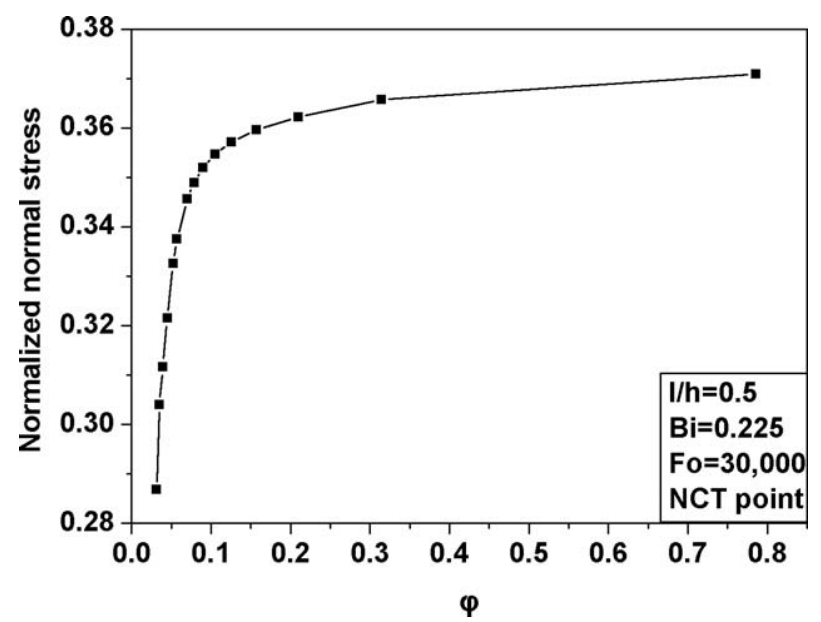

Fig. 12. The normalized NCT interfacial normal stress versus central angle of the representative sector. $l / h=0.5, \mathrm{Bi}=0.225, \mathrm{Fo}=30,000$. Other inputs are listed in Table 1. 


\section{Conclusions}

In the present paper, the problem of a multi-cracked and coated hollow cylinder, subjected to convective heating from inner surface is considered. The interfacial normal stress near the crack tip (NCT) is found to be the dominant driving force for coating debonding initiation. Sensitivity analyses indicate the surface cracking appears very important in the variation of the NCT interfacial normal stresses. By increasing the surface crack number, the NCT interfacial normal stress becomes smaller in magnitude; while by increasing the crack depth, the variation of it is reversed. In addition, the more severe the convection heating, the earlier the coating debonding will occur, but the ultimate magnitude of NCT interfacial normal stress is not influenced by the convection severity. Other factors conducive to the reduction of NCT interfacial normal stresses for a given substrate include the increase of coating's coefficient of thermal expansion and Young's modulus.

\section{Acknowledgements}

The financial support provided by the National Natural Science Foundation of China (Grant No. 50471087, 50531060) is greatly acknowledged.

\section{References}

[1] C. Chen, B. Kuo, Eng. Fract. Mech. 49 (1994) 381

[2] R. Oliveira, X.R. Wu, Eng. Fract. Mech. 27 (1987) 185.
[3] M. Perl, A. Ashkenazi, Eng. Fract. Mech. 42 (1992) 747.

[4] H.F. Nied, F. Erdogan, J. Therm. Stress. 6 (1983) 1.

[5] R. Tang, F. Erdogan, J. Eng. Gas Turbine Power 107 (1985) 212.

[6] F. Delale, S.P. Kolluri, J. Therm. Stress. 8 (1985) 235.

[7] B.D. Choules, K. Kokoni, T.A. Taylor, Surf. Coat. Technol. 106 (1998) 23.

[8] M.Y. He, J.W. Hutchinson, A.G. Evans, Mater. Sci. Eng., A Struct. Mater.: Prop. Microstruct. Process. 345 (2003) 172.

[9] D.L. Ruckle, Thin Solid Films 73 (1980) 455.

[10] T.A. Taylor, D.L. Appleby, A.E. Weatherill, J. Griffiths, Surf. Coat. Technol. 43-44 (1990) 470.

[11] P.J. Cote, C. Richard, Wear 241 (2000) 17.

[12] J.H. Underwood, A.P. Park, G.N. Vigllante, P.J. Cote, J. Press. Vessel Technol. 125 (2003) 299

[13] J.W. Hutchinson, Z. Suo, Adv. Appl. Mech. 29 (1992) 63.

[14] J.W. Hutchinson, J. Mech. Phys. Solids 49 (2001) 1847.

[15] Z. Suo, ASME J. Appl. Mech. 71 (2004) 646.

[16] Y. Hu, K. Zhang, G.N. Chen, C.W. Wu, J. Metal. Treatment Supp. 30 (2005) 161 (in Chinese).

[17] K. Zhang, C.W. Wu, Y. Hu, G.N. Chen, in: I.S. Chung (Ed.), The 3rd Asian Conference on Heat Treatment of Materials, Gyeongiu(Korea), 2005, p. 216

[18] T. Atarashi, S. Minagawa, Int. J. Eng. Sci. 30 (1992) 1543.

[19] E. Sternberg, J.G. Chakravorty, ASME J. Appl. Mech. 26 (1959) 503.

[20] G.I. Barenblatt, Scaling, Self-Similarity, and Intermediate Asymptotics, Cambridge University Press, Cambridge, 1996.

[21] Q.F. Ma, R.S. Fang, L.C. Xiang, S. Guo, Practical Handbook of Thermophysical Properties, Chinese Agricultural Press, Beijing, 1986 (in Chinese).

[22] K. Kokini, A. Banerjee, T.A. Taylor, Mater. Sci. Eng., A Struct. Mater.: Prop. Microstruct. Process. 323 (2002) 70.

[23] B. Zhou, K. Kokini, Surf. Coat. Technol. 187 (2004) 17.

[24] B. Zhou, K. Kokini, Acta Mater. 52 (2004) 4189. 